\title{
Phosphoketolase overexpression increases biomass and lipid yield from methane in an obligate methanotrophic biocatalyst.
}

\author{
Calvin A. Henard, Holly K. Smith, and Michael T. Guarnieri* \\ National Bioenergy Center, National Renewable Energy Laboratory, 15013 Denver \\ West Parkway, Golden, CO 80401 \\ *Address correspondence to: Michael T. Guarnieri, 15013 Denver West Parkway, MS \\ 3323, Golden, C0 80401, Ph: 303-384-7921. Email: Michael.Guarnieri@nrel.gov
}

\begin{abstract}
Microbial conversion of methane to high-value bio-based chemicals and materials offers a path to mitigate GHG emissions and valorize this abundant-yet underutilized carbon source. In addition to fermentation optimization strategies, rational methanotrophic bacterial strain engineering offers a means to reach industrially relevant titers, carbon yields, and productivities of target products. The phosphoketolase pathway functions in heterofermentative bacteria where carbon flux through two sugar catabolic pathways to mixed acids (lactic acid and acetic acid) increases cellular ATP production. Importantly, this pathway also serves as an alternative route to produce acetyl-CoA that bypasses the $\mathrm{CO}_{2}$ lost through pyruvate decarboxylation in the Embden-Meyerhof-Parnas pathway. Thus, the phosphoketolase pathway can be leveraged for carbon efficient biocatalysis to acetyl-CoA-derived intermediates and products. Here, we show that the industrially promising methane biocatalyst, Methylomicrobium buryatense, encodes two phosphoketolase isoforms that are expressed in methanol- and methane-grown cells. Overexpression of the PktB isoform led to a 2 -fold increase in intracellular acetyl-CoA concentration, and a 2.6-fold yield enhancement from methane to microbial biomass and lipids compared to wild-type, increasing the potential for methanotroph lipid-based fuel production. Off-gas analysis and metabolite profiling indicated that global metabolic rearrangements, including significant increases in post-translational protein acetylation and gene expression of the tetrahydromethanopterin-linked pathway, along with decreases in several excreted products, coincided with the superior biomass and lipid yield observed in the engineered strain. Further, these data suggest that phosphoketolase may play a key regulatory role in methanotrophic bacterial metabolism. Given that acetyl-CoA is a key intermediate in several biosynthetic pathways, phosphoketolase overexpression offers a viable strategy to enhance the economics of an array of biological methane conversion processes.
\end{abstract}

Keywords: methane biocatalysis, methanotroph, phosphoketolase, biogas, greenhouse gas mitigation 


\section{Introduction}

Methane $\left(\mathrm{CH}_{4}\right)$ is the second most abundant greenhouse gas and has significantly more global warming potential compared to the most abundant greenhouse gas, carbon dioxide. $\mathrm{CH}_{4}$ emissions are largely produced from anthropogenic sources, being a primary component of natural gas and anaerobic digestion-derived biogas. In addition, $\mathrm{CH}_{4}$ discharged from ruminant livestock and landfills further contribute to global $\mathrm{CH}_{4}$ emissions ${ }^{1,2}$. Rising atmospheric $\mathrm{CH}_{4}$ levels necessitate the development of novel technologies to mitigate GHG emissions. Methane biocatalysis using natural $\mathrm{CH}_{4}$-consuming bacteria (methanotrophs) offers a scalable and selective route to utilize an overabundant, often wasted carbon source, while also mitigating greenhouse gas emissions ${ }^{3,4}$.

Methanotrophs use $\mathrm{CH}_{4}$ as their sole carbon and energy source, and play a key role in the biogeochemical cycle by serving as a $\mathrm{CH}_{4} \operatorname{sink}^{5,6}$. These organisms hold great promise for the capture and conversion of methane from stranded natural gas, anaerobic-digestion-derived biogas, and other anthropogenic $\mathrm{CH}_{4}$ sources, but are currently limited by poor product titers, yields, and productivities associated with inefficient microbial methane utilization and inefficient carbon conversion $7,84,9$. Recent development of genetic tools for use in industrially relevant methanotrophs has facilitated rational strain engineering of these organisms for the conversion of $\mathrm{CH}_{4}$ to fuels and chemicals ${ }^{8,10-12}$. Leveraging these tools to develop a biocatalyst with increased methane conversion efficiency would dramatically enhance process economics, allowing $\mathrm{CH}_{4}$ bioconversion to be competitive with conventional sugar upgrading processes.

Phosphoketolases (PKTs) have been used for engineering carbon-efficient processes from sugars due to their intrinsic ability to bypass glycolytic pyruvate decarboxylation ${ }^{13}$. These enzymes catalyze the irreversible cleavage of xylulose-5phosphate and/or fructose-6-phosphate to acetyl-phosphate (acetyl-P) and glyceraldehyde-3-phosphate or erythrose-4-phosphate, respectively ${ }^{14,15}$. The acetyl$\mathrm{P}$ produced by PKT is readily converted to acetyl-CoA with complete carbon conservation (Figure 1). Although PKT engineering strategies offer the advantage of complete carbon conversion, this occurs at the expense of reducing equivalents and ATP generated in downstream glycolytic biochemical conversions that are bypassed by the PKT pathway. PKTs have been characterized in obligate and heterofermentative bacteria and some species of fungi where they enable an alternative pathway for sugar-phosphate degradation. Interestingly, PKT orthologs have been observed in the genomes of an array of C1-utilizing microbes ${ }^{16}$, and it has been postulated that the PKT pathway may serve an important metabolic role in methanotrophic, methylotrophic, and autotrophic bacteria ${ }^{16,17}$.

PKT pathway engineering has been successfully used to increase yield of an array of acetyl-CoA-derived products in diverse microbial biocatalysts, including 
Corynebacterium glutamicum ${ }^{18}$, Saccharomyces cerevisiae ${ }^{13,19-23}$. Aspergillus nidulans ${ }^{24}$ and Escherichia coli ${ }^{25}$ by providing a larger pool of acetyl-CoA precursor; however, they have yet to be leveraged to improve $\mathrm{CH}_{4}$ biocatalysis. Herein, we overexpressed native methanotroph PKTs as a strategy to increase methane conversion efficiency to biomass in an obligate methanotrophic bacterium. Overexpression of the PktB isoform in the gammaproteobacterial methanotroph, Methylomicrobium buryatense, resulted in global metabolic alterations, ultimately increasing the biomass and lipid yield from $\mathrm{CH}_{4}$ by 2.6- and 2-fold, respectively, compared to wild-type in the engineered strain. These results represent the most significant yield improvement via PKT pathway engineering in any organism reported to date, and also support a novel function for PKT in methanotroph posttranslational regulation.

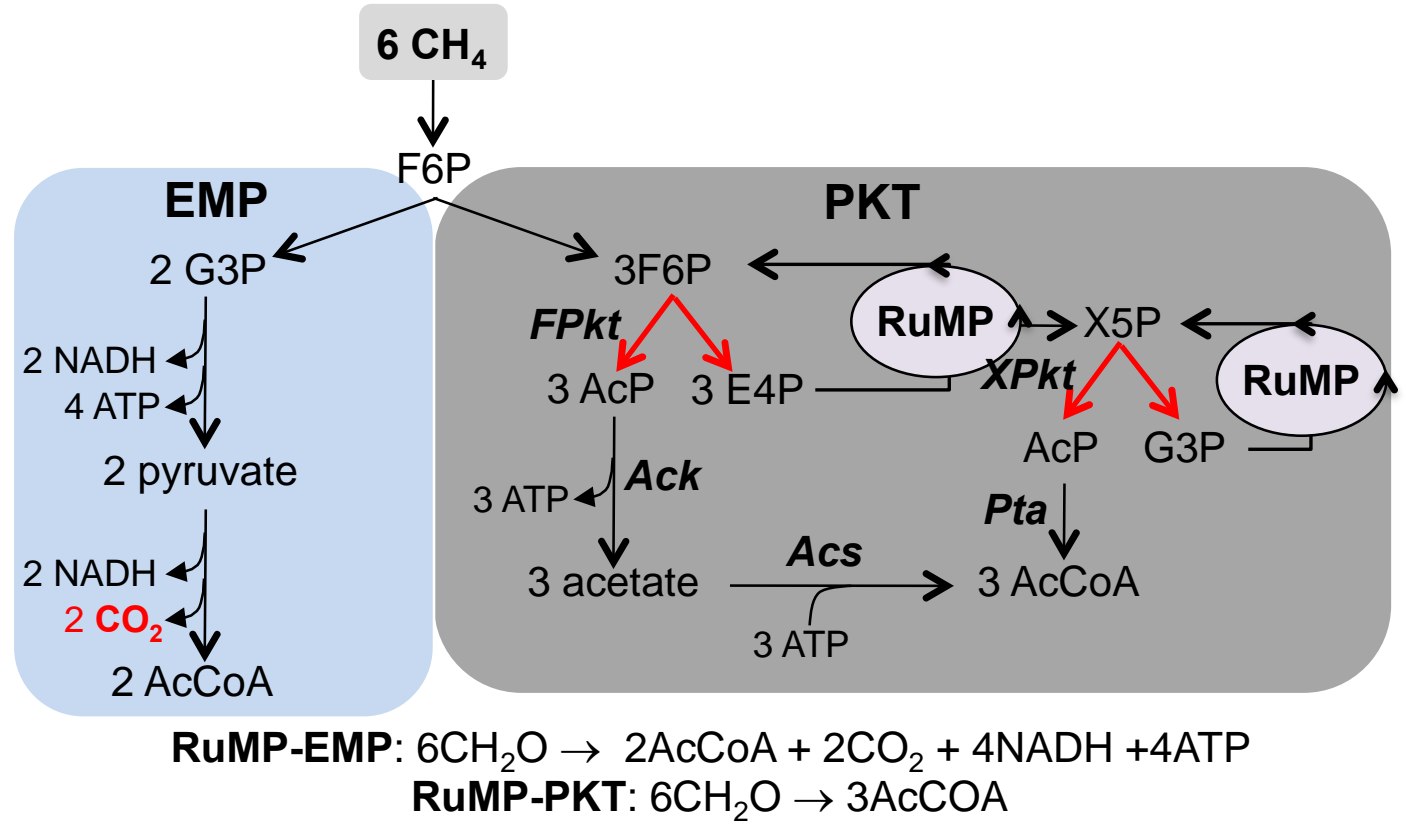

Figure 1. Carbon flux through the PKT pathway bypasses pyruvate decarboxylation. $\mathrm{CH}_{4}$ is assimilated to fructose-6-phosphate (F6P) in gammaproteobacterial methanotrophs, which enters the Embden-Meyerhof-Parnas (EMP) pathway. F6P and/or xylulose-5-phosphate (X5P) can be converted to intermediates of the ribulose phosphate (RuMP) pathway and acetyl-P (AcP) by phosphoketolase (PKT, red arrows). AcP is readily converted to acetyl-CoA (AcCoA) by the phosphotrasacetylase (Pta), bypassing the $\mathrm{CO}_{2}$ lost in the decarboxylation of pyruvate to AcCoA. FPkt, F6P phosphoketolase; XPkt, X5P phosphoketolase; G3P, glyceraldehyde-3-phosphate; E4P, erythrose4-phosphate; Ack, acetate kinase; Acs, acetyl-CoA synthase. Adapted from ${ }^{13}$

\section{Materials and methods}

\subsection{Microbial strains and cultivation}

Methylomicrobium buryatense $5 \mathrm{~GB}^{\mathrm{S}} \mathrm{S}^{10}$ and engineered strains (Table S1) were routinely cultivated in NMS2 medium at $30^{\circ} \mathrm{C}$ with orbital shaking at $225 \mathrm{rpm}$ as 
previously described ${ }^{8}$. Strains were grown in sealed $150 \mathrm{~mL}$ glass serum bottles (Kimble Chase, Vineland, NJ) with $20 \%$ (v/v) $\mathrm{CH}_{4}$ in air, or $500 \mathrm{~mL}$ baffled flasks supplemented with $1 \% \mathrm{CH}_{3} \mathrm{OH}(\mathrm{v} / \mathrm{v}$ ). Escherichia coli Zymo 5a (Zymo Research, Irvine, $\mathrm{CA}$ ) was used for cloning and plasmid propagation. Polymerase chain reactions were performed using Q5 High-Fidelity Polymerase from New England Biolabs and primers (Table S2) purchased from Integrated DNA Technologies (Coralville, IO).

\subsection{Plasmid construction}

Plasmids for constitutive phosphoketolase expression were constructed by ligating the methanol dehydrogenase promoter region $\left(\mathrm{P}_{m x a}\right)$, pktA (METBUDDRAFT_0184) or pktB (METBUDRAFT_1551), and the Escherichia coli rrnBT1T2 terminator into the pAWP78 IncP-based vector ${ }^{10}$ to generate pPKTA and pPKTB using 2X Gibson Assembly Mix from New England Biolabs (Ipswich, MA) following the manufacturers protocol. The pPKTA and pPKTB vectors were confirmed by sequence analysis (Genewiz, South Plainfield, NJ). These vectors were transformed into $M$. buryatense via biparental mating using $E$. coli $\mathrm{S} 17-1$ as previously described ${ }^{8}$. Positive transformants selected on NMS2 agar containing $50 \mu \mathrm{g} / \mathrm{mL}$ of kanamycin were confirmed using primers oCAH042 and oCAH043 in polymerase chain reactions.

\subsection{Real-time PCR}

Total RNA was isolated from logarithmically growing cells $\left(\mathrm{OD}_{600}=1.0\right.$ using TriZol reagent (ThermoFisher) followed by removal of contaminating DNA by performing treatment with Turbo DNase (Life Technologies, Carlsbad, CA. cDNA was synthesized from $1 \mu \mathrm{g}$ total RNA using iScript reverse transcriptase (Biorad, Hercules, CA) following the manufacturer's protocol. Real-time PCR was performed by using SYBR IQ master mix and a BioRad iCyler iQ Real-Time PCR System (BioRad). The primers used for the real-time RT-PCR are listed in Table S2. The cycle threshold $(\mathrm{Ct}$ ) value for each gene was determined and normalized to the rpoD housekeeping sigma factor gene. Relative expression between two samples was calculated by using the $\Delta \Delta \mathrm{Ct}$ method ${ }^{24}$. Growth in $\mathrm{CH}_{3} \mathrm{OH}$ or $\mathrm{CH}_{4}$ as the carbon source did not result in any significant changes $(P>0.05)$ in rpoD mRNA expression.

\subsection{SDS-PAGE}

Cell-free extracts were prepared by sonication of logarithmically growing $(20 \%$ $\mathrm{CH}_{4}$ ) wild-type and engineered strains of $M$. buryatense in sample buffer $(50 \mathrm{mM}$ Tris pH 8.0 containing $100 \mathrm{mM} \mathrm{NaCl}$ ). Protein concentrations in clarified extracts were determined with the Pierce 660nm Protein Assay Reagent (Life Technologies) using bovine serum albumin as a protein standard and normalized by the addition of sample buffer. Samples were mixed with $3 X$ Red loading buffer (New England Biolabs, Ipswich, MA) containing $2 \mathrm{mM}$ 2-mercaptoethanol. $5 \mu \mathrm{g}$ total protein was loaded into an Any kD Mini-PROTEAN TGX Precast protein gel (Biorad) and electrophoresed at 150V. Proteins were visualized by Coomassie blue staining. 


\subsection{Phosphoketolase enzyme assay}

Phosphoketolase activity in whole-cell lysates prepared from logarithmically growing wild-type $\left(20 \% \mathrm{CH}_{4}\right)$, pPKTA, and pPKTB strains was measured using a hydroxymate assay that detects acetyl phosphate ${ }^{26,27}$. Briefly, cells $\left(\mathrm{OD}_{600}=1.0\right)$ were pelleted, resuspended in $50 \mathrm{mM}$ potassium phosphate buffer ( $\mathrm{pH} 6.5$ ) and disrupted by sonication on ice. $100 \mu \mathrm{L}$ reactions in reaction buffer $[30 \mathrm{mM}$ potassium phosphate ( $\mathrm{pH} 6.5$ ), L-cysteine hydrochloride ( $2 \mathrm{mM}$ ), sodium fluoride $(20 \mathrm{mM})$, sodium iodoacetate $(10 \mathrm{mM})$, thiamine pyrophosphate $(1 \mathrm{mM})$, and fructose 6-phosphate (30 mM) (Sigma)] were initiated by the addition of $25 \mu \mathrm{L}(10$ $\mu \mathrm{g}$ total protein) of whole-cell extracts. Reactions were incubated at $37^{\circ} \mathrm{C}$ for $1 \mathrm{~h}$. After incubation, $50 \mu \mathrm{L}$ of $2 \mathrm{M}$ hydroxylamine hydrochloride ( $\mathrm{pH} 7.0$ ) was added to the reaction and incubated at room temperature for $10 \mathrm{~min}$. Reactions were then terminated by adding $300 \mu \mathrm{L}$ of a 1:1 mixture of $2.5 \%$ ferric chloride in $2 \mathrm{M}$ hydrochloric acid and $10 \%$ trichloroacetic acid. A color change due to ferrichydroxamate formation was measured at $\mathrm{A}_{505 \mathrm{~nm}}$, and the concentration of acetyl-P formed during the initial reaction was calculated by regression analysis compared to known standards.

\section{$2.6 \mathrm{CH}_{4}$ fermentations}

Seed cultures were started from plate biomass and grown in $1 \mathrm{~L}$ flasks $\left(30^{\circ} \mathrm{C}\right.$, $500 \mathrm{rpm})$ containing NMS2 medium supplemented with $2 \mathrm{X} \mathrm{KNO}_{3}(20 \mathrm{mM})$ with a continuous gas flow $\left(20 \% \mathrm{CH}_{4}\right.$ in air). Batch $\mathrm{CH}_{4}$ fermentations were performed in a 0.5 L Biostat-Q plus bioreactor (Sartorius, Gottingen, Germany) containing NMS2 medium supplemented with $8 \mathrm{X} \mathrm{KNO}_{3}(80 \mathrm{mM}), 2 \mathrm{X}$ phosphate buffer $(4.6 \mathrm{mM})$, and $4 \mathrm{X}$ trace element solution to support high cell growth at $30^{\circ} \mathrm{C}$ as previously described $^{8}$. Seed cultures were diluted to a final $\mathrm{OD}_{600}=0.5-1.0$. Mixing was achieved by using a bottom marine impeller at $500 \mathrm{rpm}$. A continuous flow rate of $300 \mathrm{ccm} 20 \%(\mathrm{v} / \mathrm{v})$ methane in air was maintained. Culture samples were taken at the indicated times and growth was determined as $\mathrm{OD}_{600}$ or dry cell weight after freeze-drying. The percent $\mathrm{CH}_{4}$ and $\mathrm{CO}_{2}$ in the off-gas was measured every $10 \mathrm{~min}$ for the duration of bioreactor fermentations by using BlueSens gas detectors (Herten, Germany). $\mathrm{CH}_{4}$ consumption was determined by calculating the difference between off-gas detected before and after inoculation. $\% \mathrm{CH}_{4}$ consumption was converted to weight based on $\mathrm{CH}_{4}$ density $(0.000656 \mathrm{~g} / \mathrm{mL})$ and the flow rate $(60$ $\mathrm{mL} \mathrm{CH}_{4} / \mathrm{min}$ ). The percent excreted $\mathrm{CO}_{2}$ in the off-gas was converted to weight based on $\mathrm{CO}_{2}$ density $(0.00196 \mathrm{~g} / \mathrm{mL})$ and the gas flow rate $(300 \mathrm{~mL} / \mathrm{min})$.

\subsection{HPLC analysis}

HPLC was used to detect formate, acetate, and methanol in culture supernatants. At indicated intervals, growth was measured spectrophotometrically and a $1 \mathrm{~mL}$ sample was taken for HPLC analysis. The culture supernatant was filtered using a 
$0.2 \mu \mathrm{m}$ syringe filter and then a $0.1 \mathrm{~mL}$ injection was separated using a model 1260 HPLC (Agilent, Santa Clara, CA) and a cation H HPx-87H column (Bio-Rad). A $0.6 \mathrm{~mL} / \mathrm{min}$ flow rate at $55^{\circ} \mathrm{C}$ with $0.01 \mathrm{~N}$ sulfuric acid as the mobile phase was used. DAD detection was measured at $220 \mathrm{~nm}$ and referenced at $360 \mathrm{~nm}$, and metabolite concentrations were calculated by regression analysis compared to known standards.

\subsection{Intracellular Acetyl-CoA Quantification}

Intracellular concentrations of acetyl-CoA were measured by using a AcetylCoenzyme A Assay Kit (Sigma) following the manufacturers protocol. Briefly, $10 \mathrm{~mL}$ of wild-type and pPKTB cultures grown with a continuous gas flow $\left(20 \% \mathrm{CH}_{4}\right.$ in air) was pelleted and immediately frozen at $-80^{\circ} \mathrm{C}$. Cell pellets were thawed on ice and resuspended in $500 \mu \mathrm{L}$ assay buffer followed by $3 \times 30$ s rounds of sonication on ice. Clarified lysates were deproteinated by using Amicon Ultra $0.5 \mathrm{~mL} 3 \mathrm{~K}$ devices (ThermoFisher). $50 \mu \mathrm{L}$ deproteinized sample was added per well and pmol acetylCoA per well was calculated by regression analysis based on known standards. The sample data were normalized to dry cell weight by converting culture densities to dry cell weight based on culture volume and $\mathrm{OD}_{600}$ using the equation DCW $(\mathrm{g} / \mathrm{L})=$ $0.3479 * \mathrm{OD}_{600}+2.702$ developed by comparing culture density to DCW in bioreactor fermentations.

\subsection{FAME analysis}

Whole biomass lipid content was measured by gas chromatography as fatty acid methyl esters (FAMEs) after transesterification of total chloroform:methanol (2:1, $\mathrm{v} / \mathrm{v}$ )-extracted lipid, as described previously ${ }^{8}$. Detected FAME species were identified via comparison to known standards.

\subsection{Metabolite profiling}

Intracellular and excreted metabolites were analyzed by Metabolon, Inc. (Durham, $\mathrm{NC}$ ) from cultures grown in $1 \mathrm{~L}$ flasks $\left(30^{\circ} \mathrm{C}, 500 \mathrm{rpm}\right)$ with a continuous gas flow $\left(20 \% \mathrm{CH}_{4}\right.$ in air $)$. At $\mathrm{OD}_{600}=1.0$, cultures were pelleted at $4300 \mathrm{rpm}$ for $5 \mathrm{~min}$ at $4{ }^{\circ} \mathrm{C}$. Culture supernatant and pelleted biomass were then transferred to $2.0 \mathrm{~mL}$ screwtop vials and flash frozen in liquid nitrogen. Sample analyses were conducted as described previously ${ }^{28}$. In brief, samples were extracted and split into equal parts for analysis on the LC/MS/MS, and Polar LC platforms. Ions were matched to an inhouse library of standards for metabolite identification and for metabolite quantitation by peak area integration. Welch's two-sample $t$-tests and Principal Component Analysis (PCA) were used to analyze the data. For all analyses, following normalization to protein measured by Bradford, missing values, if any, were imputed with the observed minimum for that particular compound. The statistical analyses were performed on natural log-transformed data and were considered significant if $p<0.05$. 


\section{Results and Discussion}

\subsection{Methylomicrobium buryatense expresses two phosphoketolase isoforms.}

PKT orthologs have been observed in the genomes of an array of methanotrophic and methylotrophic bacteria ${ }^{16}$; however, their role in $\mathrm{CH}_{4}$ metabolism remains elusive. M. buryatense encodes two putative PKT isoforms, PktA and PktB, that share $63.7 \%$ amino acid identity (Figure S1). To establish baseline expression of these isoforms, we evaluated transcription of the native PKTs and associated pathway genes during growth on either $\mathrm{CH}_{4}$ or $\mathrm{CH}_{3} \mathrm{OH}$. Real-time PCR analysis indicated that $M$. buryatense expresses a complete phosphoketolase pathway, including $p k t A, p k t B$, acetate kinase $a c k$, phosphotransacetylase $p t a$, and the acetyl-CoA synthase acs genes, when grown with $\mathrm{CH}_{3} \mathrm{OH}$ or $\mathrm{CH}_{4}$ as the sole carbon source (Figure S2). Interestingly, the PKT pathway genes were highly upregulated (100-1000 fold) in $\mathrm{CH}_{4}$-grown cells compared to $\mathrm{CH}_{3} \mathrm{OH}$-grown cells (Figure 2). These data provide support for an alternative route of carbon flux in $\mathrm{CH}_{4}$-grown $M$. buryatense, which should be considered in future metabolic models and flux analyses. Additionally, the conserved nature of the PKT pathway in an array of methanotrophic, methylotrophic, and autotrophic organisms supports that this pathway likely plays an important role in $\mathrm{C} 1$ metabolism.

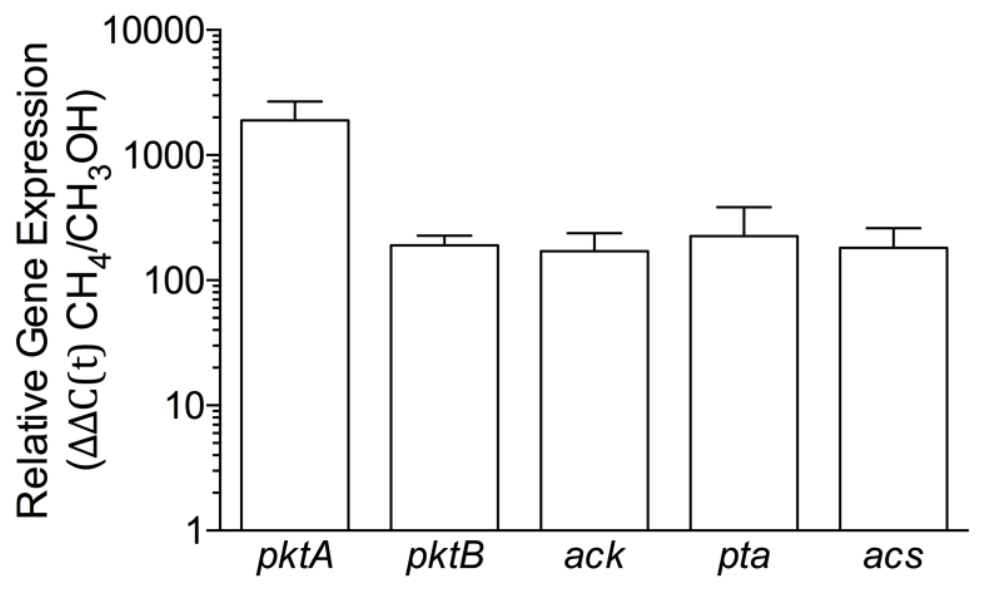

Figure 2. Methylomicrobium buryatenese expresses a complete phosphoketolase pathway. Real-time PCR analysis of relative phosphoketolase pathway transcription comparing M. buryatense grown with $\mathrm{CH}_{4}$ or $\mathrm{CH}_{3} \mathrm{OH}$ as the carbon source. pktA (phosphoketase isoform $\mathrm{A}$ ), pktB (phosphoketolase isoform B), ack (acetate kinase), pta (phosphotransacetylase), acs (acetyl-CoA synthase). Data represent the mean \pm SD of 3 biological replicates.

\subsection{Phosphoketolase pathway overexpression in M. buryatense.}

The phosphoketolase pathway has been leveraged to enhance metabolic processes from sugars in several microbial biocatalysts ${ }^{13}$ and from $\mathrm{CH}_{3} \mathrm{OH}$ in a synthetic in vitro pathway ${ }^{29}$, but has yet to be explored in $\mathrm{CH}_{4}$ bioconversion processes. Given that M. buryatense expresses all PKT pathway components, we examined overexpression of the PKT isoforms in order to achieve high carbon flux through the 
pathway. PktA and PktB isoforms were overexpressed using an IncP-based vector, driven by the methanol dehydrogenase $\left(\mathrm{P}_{m \times a}\right)$ promoter (Figure $3 \mathrm{~A}$ ), one of the most active promoters in Methylomicrobium species ${ }^{30}$. These PKT expression vectors were conjugated into $M$. buryatense through biparental mating to generate the engineered pPKTA and pPKTB strains. Both strains exhibited significantly enhanced transcription and protein content of their respective $\mathrm{PKT}$ isoform during $\mathrm{CH}_{4}$ dependent growth (Figure 3B and C), with an associated increase in PKT activity compared to wild-type M. buryatense (Figure 3D). Interestingly, although expression of the PKT isoforms was independently under the control of the $\mathrm{P}_{m \times a}$ promoter, we observed higher transcription, protein expression, and associated PKT activity in the pPKTA strain, potentially due to differential post-transcriptional regulation between the two PKT isoforms. Growth kinetics were similar between wild-type and pPKTB, which both reached high cell density $0_{600} \cong 20$ (Figure $4 \mathrm{~A}$ ). However, the pPKTA strain exhibited a growth defect after $48 \mathrm{~h}$ (Figure 4B), presumably due to either the high concentrations of acetate excreted into the medium or the increased PktA expression by this strain ${ }^{31}$. Given that PktA overexpression caused growth inhibition, we focused on the pPKTB strain for the remainder of our studies. The pPKTB strain displayed a 2-fold increase in intracellular acetyl-CoA concentration. This, coupled with the observed increase in acetate excretion (Figure 4B), indicates significant flux through the PKT pathway is occurring in this strain.

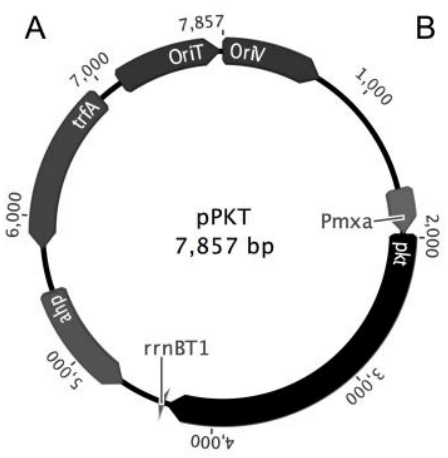

B
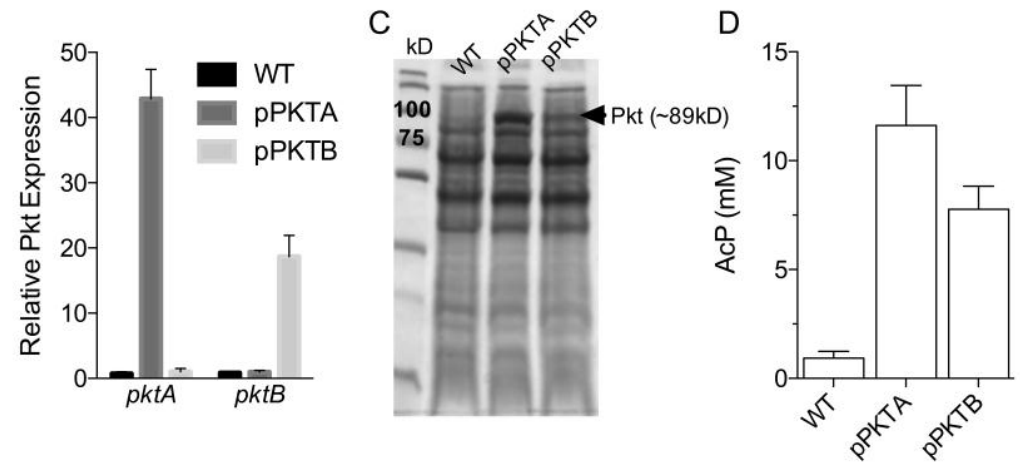

Figure 3. Overexpression of the native phosphoketolases in M. buryatense. A) Plasmids were constructed for high, constitutive expression of two PKT isoforms (PktA and PktB) under the control of the methanol dehydrogenase promoter $\left(P_{m x a}\right)$. B) Real-time PCR analysis and C) SDS-PAGE analysis of whole-cell lysates confirming increased PKT transcription and protein expression by engineered strains, respectively. D) In vitro conversion of fructose-6-phosphate to acetyl-phosphate (AcP) by whole-cell lysates determined by using the hydroxymate method. Data in B and D represent the mean \pm SD of 3 biological replicates. 

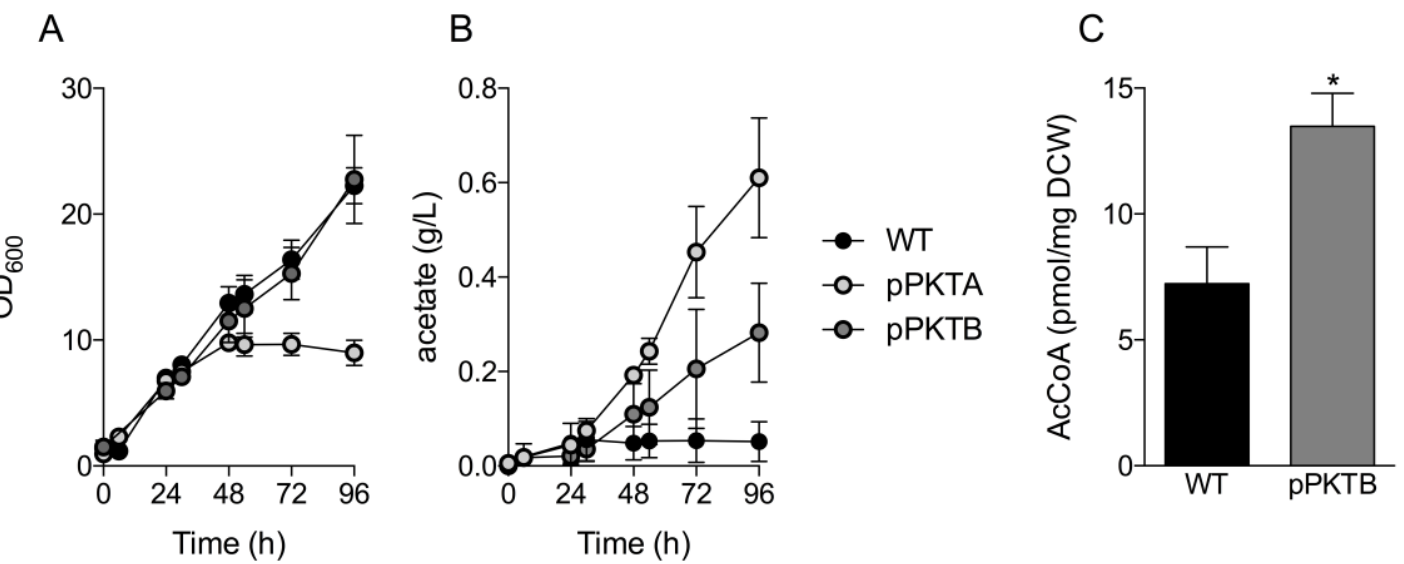

Figure 4. Increased production of acetate and acetyl-CoA in phosphoketolase overexpressing strains. A) Growth and B) excreted acetate by wild-type (black circles) and engineered M. buryatense overexpressing PktA (light grey circles) or PktB (grey circles). C) Comparison of intracellular acetylcoenzyme A (AcCoA) concentration in wild-type (black bar) and pPKTB (grey bar). Data represents the mean \pm SD of $2-3$ biological replicates. ${ }^{*} p<0.05$.

\subsection{Phosphoketolase overexpression increases biomass and lipid yield in bioreactor fermentations.}

In stirred-tank bioreactors, pPKTB grew to higher cell density (10.3 g/L DCW) compared to wild-type (7.9 g/L DCW) after $96 \mathrm{~h}$ of growth (Figure $5 \mathrm{~A})$. Wild-type $M$. buryatense consumed $\mathrm{CH}_{4}$ at a rate $(0.063 \mathrm{~g} / \mathrm{h}$, Figure $5 \mathrm{~B}$ and $\mathrm{C})$ comparable to that previously reported for this organism ${ }^{7}$. Interestingly, despite superior biomass accumulation by pPKTB, its $\mathrm{CH}_{4}$ assimilation rate was much lower $(0.034 \mathrm{~g} / \mathrm{h}$, Figure 5B), significantly $(p<0.01)$ improving the biomass yield in this strain by 2.6 fold (Figure 5C; pPKTB, $1.1 \mathrm{~g}$ DCW/g CH 4 vs wild-type, $0.43 \mathrm{~g}$ DCW/g CH 4 ). A similar increase in pPKTB biomass yield was also observed in shake-flask experiments with $1 \% \mathrm{CH}_{3} \mathrm{OH}$ as the sole carbon source (Figure S3).

In order to accommodate the particulate methane monoxygenase responsible for methane activation, methanotrophs synthesize an elaborate intracellular membrane network that is a viable fuel molecule precursor ${ }^{12}$. Given that we observed a 2-fold increase in lipid precursor acetyl-CoA levels, we evaluated the lipid composition of wild-type and pPKTB. Total lipid content was similar between the two strains ( $9 \%$ based on DCW, Figure 5D), which primarily consisted of hexadecanoic and hexadecenoic acids (Table S3), resulting in $\sim 2$-fold improvement in lipid yield (Figure 5E). 

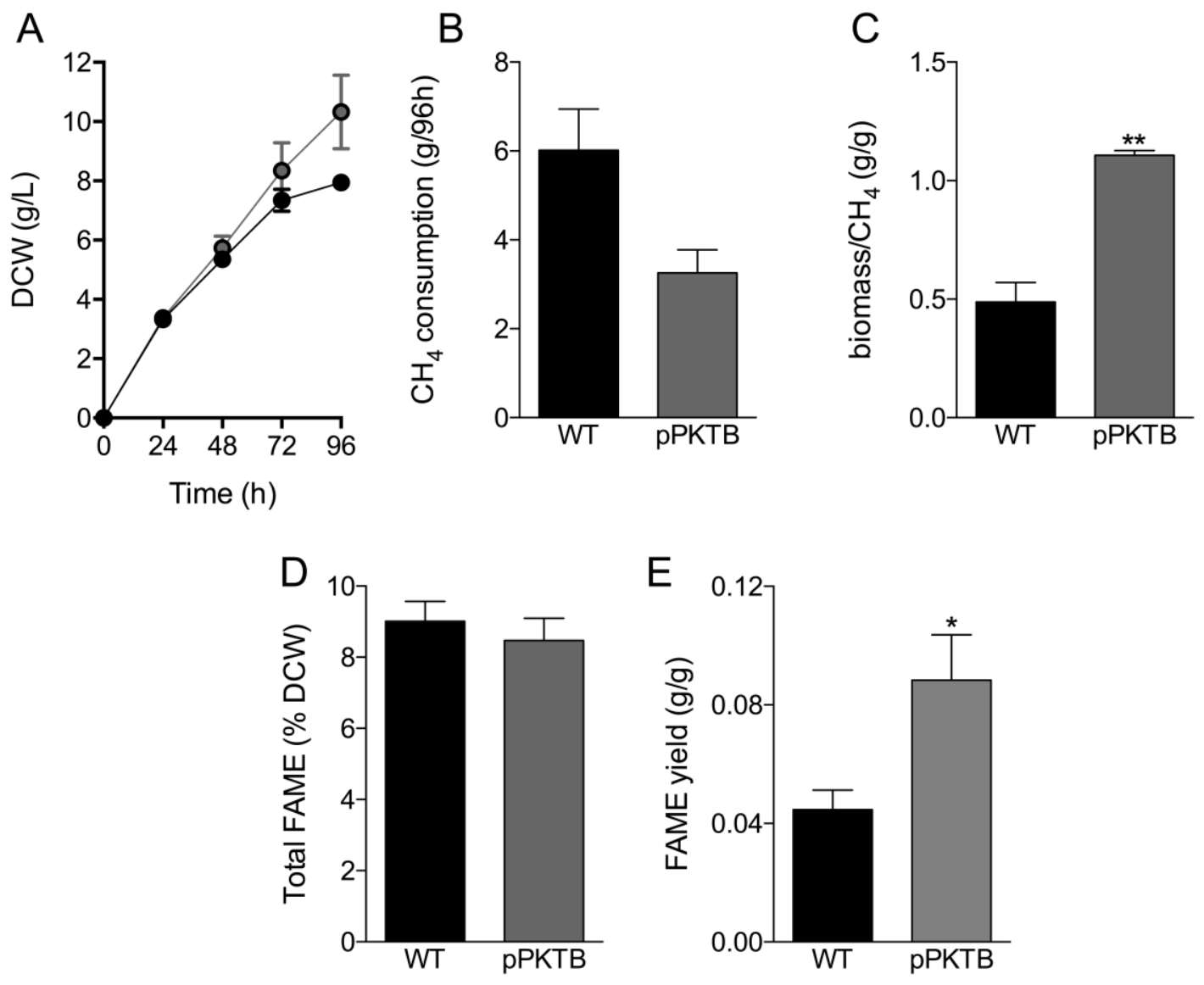

Figure 5. Phosphoketolase overexpression increases methanotroph biomass and lipid yield from methane. A) Growth (DCW, dry cell weight) of wild-type (black circles/bars) and engineered (grey circles/bars) M. buryatense was measured over time in a $0.5 \mathrm{~L}$ gas bioreactor with continuous $\mathrm{CH}_{4}$ feed (20\% $\mathrm{CH}_{4}$ in air). B) Total $\mathrm{CH}_{4}$ consumed during $96 \mathrm{~h}$ of culture. C) Ratio of biomass DCW to total $\mathrm{CH}_{4}$ consumption after $96 \mathrm{~h}$. D) Total lipid content presented as fatty acid methyl esters (FAME) of wild-type (black bars) and engineered pPKTB (grey bars) M. buryatense dry cell weight (DCW). E) Ratio of FAME titer to total $\mathrm{CH}_{4}$ consumption after $96 \mathrm{~h}$. Data represent the mean $\pm \mathrm{SD}$ of 2-3 biological replicates. ${ }^{*} p<0.05,{ }^{* *} p<0.01 .$.

\subsection{Metabolite profiling reveals carbon flux alterations in the engineered methanotrophic strain.}

Methanotrophs convert $\mathrm{CH}_{4}$ to $\mathrm{CH}_{3} \mathrm{OH}$, which is further oxidized to formaldehyde $\left(\mathrm{CH}_{2} \mathrm{O}\right)$. In gammaproteobacterial methanotrophs like $M$. buryatense, $\mathrm{CH}_{2} \mathrm{O}$ is either assimilated into central carbon metabolism or further oxidized to formate $(\mathrm{CHOOH})$ and $\mathrm{CO}_{2}$ to produce $\mathrm{NADH}$ reducing equivalents via the tetrahydromethanopterin $\left(\mathrm{H}_{4} \mathrm{MPT}\right)$-linked dissimilatory pathway ${ }^{30,32}$. A recent in silico metabolic flux model ${ }^{33}$ suggests that complete carbon flux through the PKT pathway would increase $\mathrm{CH}_{4}$ oxidation to $\mathrm{CO}_{2}$ in order to supply NADH and compensate for the reducing 
equivalents lost at the expense of increased flux through the redox-neutral PKT pathway (Figure 1). To test this hypothesis, we evaluated expression of the $\mathrm{H}_{4} \mathrm{MPT}$ linked formaldehyde activating enzyme fae and formate dehydrogenase $f d h$ genes. As shown in Figure 6A, transcription of all fae and fdh genes was increased in the pPKTB strain compared to wild-type. Complete oxidation of $\mathrm{CH}_{4}$ through the $\mathrm{H}_{4} \mathrm{MPT}$ pathway is supported by a significant decrease in excreted formate by pPKTB (Figure 6B), which, instead of being excreted, is likely oxidized to $\mathrm{CO}_{2}$ due to the increase in NAD-linked formate dehydrogenase expression in this strain. We next examined the potential impact of PKT overexpression on $\mathrm{CO}_{2}$ evolution. We observed decreased $\mathrm{CO}_{2}$ evolution from the pPKTB culture (Figure S4), but the ratio of evolved $\mathrm{CO}_{2}$ to assimilated $\mathrm{CH}_{4}$ was not significantly altered in pPKTB compared to wild-type in stirred-tank bioreactors (data not shown). In agreement with the metabolic flux model that PKT overexpression increases reducing equivalent production via the dissimilatory pathway 33 , our data indicate that carbon typically excreted by wild-type cells is, instead, oxidized to $\mathrm{CO}_{2}$ in an effort to produce NAD(P)H. However, in contrast to the model, PKT overexpression does not divert $\mathrm{CH}_{4}$ flux through the dissimilatory pathway away from biomass. PKT overexpression resulting in increased biomass yield suggest this strain not only is more efficient at utilizing assimilated carbon, but may also possess increased energy efficiency mediated through unknown mechanisms.

A

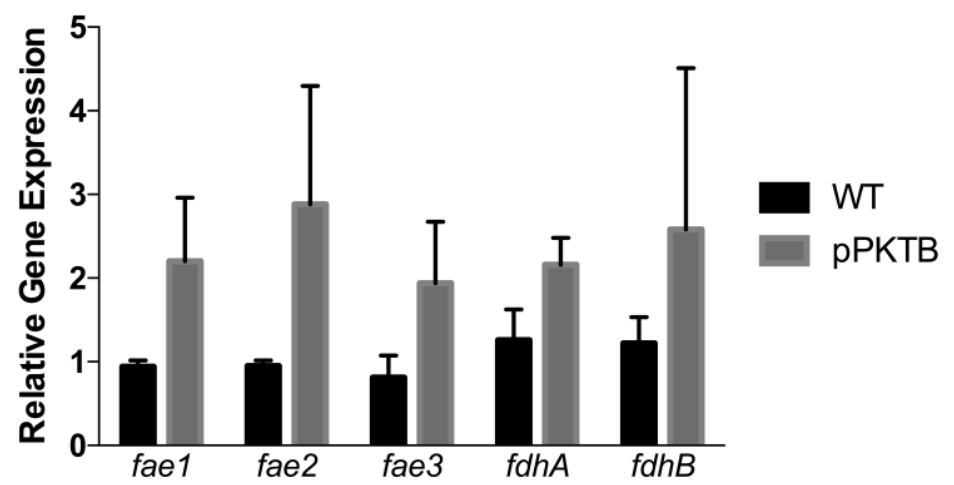

B

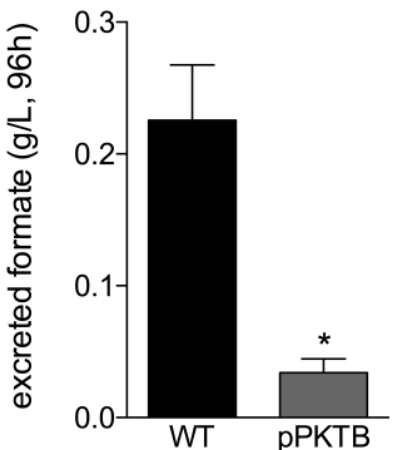

Figure 6. PKT overexpression decreases flux through the tetrahydromethopterin $\left(\mathrm{H}_{4} \mathrm{MPT}\right)$ linked C1 transfer pathway. A) Real-time PCR analysis of $\mathrm{H}_{4}$ MPT pathway gene transcription in wild-type (black bars) and engineered (grey bars) strains. B) Excreted formate in culture supernatants detected by HPLC. fae (formaldehyde activating enzyme), $f d h$ (formate dehydrogenase). Data represent the mean $\pm S D$ of at least 3 biological replicates. ${ }^{*} p<0.05$.

To gain further insight into the increase in biomass yield, we examined intracellular and excreted metabolite profiles in wild-type and pPKTB $M$. buryatense actively growing on $\mathrm{CH}_{4}$ (Figure 7). The pPKTB strain had several significantly increased protein acetylation markers, with a 46-fold increase in intracellular N-6-acetyllysine compared to wild-type cells (Table S3). These data further support that the pPKTB strain has significantly elevated levels of intracellular acetyl-CoA, which is the primary substrate for $\mathrm{N}$-acetyl transferase-dependent acetylation. In addition, the CoA precursor, panthothenic acid was significantly increased in pPKTB, which has 
been shown to increase intracellular acetyl-CoA levels when supplemented in E. coli growth medium ${ }^{34}$. Other notable intracellular metabolites significantly altered in the pPKTB strain included the PKT cofactor thiamine diphosphate, sedoheptulose of the pentose phosphate pathway, and pyruvate. Further, nicotinic acid was 50 -fold higher in pPKTB cells compared to wild-type, potentially due to increased de novo synthesis of pyridine nucleotides to overcome the limited reducing power in this strain. Compared to wild-type, several metabolites were found to be decreased in the culture supernatants of the pPKTB strain, including malate and succinate of the TCA cycle and xanthine/hypoxanthine of purine metabolism (Table S4). In contrast, both glutamate and glutamine were significantly higher in the pPKTB supernatant compared to wild-type, implicating a role for PKT in nitrogen assimilation.

A

intracellular metabolites (pPKTBNT)

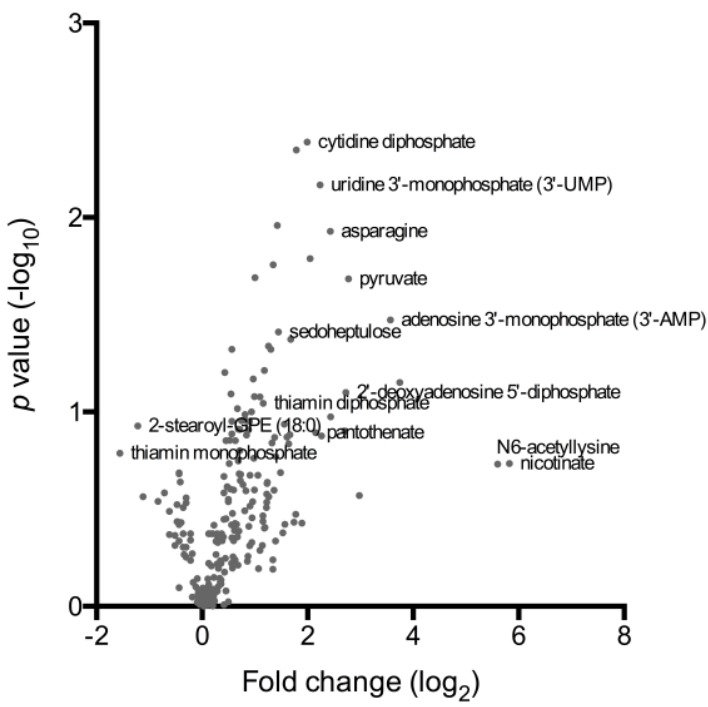

excreted metabolites (pPKTBMT)

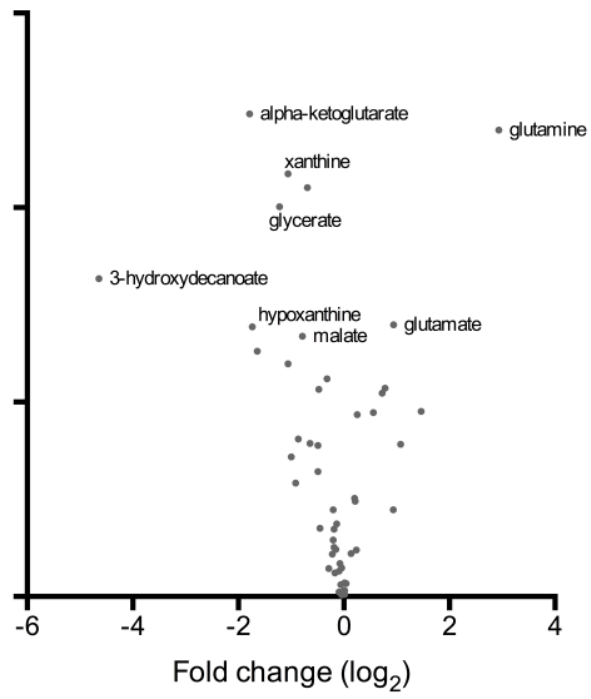

Figure 7. Phosphoketolase overexpression results in alterations to both intracellular and excreted metabolites. Positively and negatively altered A)intracellular and B)excreted metabolites are presented as a volcano plot with $p$-value vs. the metabolite ratio of pPKTB to wild-type (WT) during growth on a continuous $20 \% \mathrm{CH}_{4}$ in air gas stream.

In addition to biomass enhancements directly related to increased acetyl-CoA, a number of additional pathways could be altered in the pPKTB strain due to global regulatory alterations. For example, aerobic methanotrophs excrete a substantial amount of carbon as exopolymeric substance, which can account for upwards of $30 \%(\mathrm{~g} / \mathrm{g})$ of total assimilated carbon ${ }^{35}$. Quantification of EPS and other excreted products not identified by metabolomics in this study may provide further insight into the redistribution of cellular carbon that resulted in the increased pPKTB biomass yield. We note that global metabolomic analyses indicate decreased abundance of several key metabolites, including formate, malate, and succinate. Thus, it is feasible that alterations in biomass yield are due to additive effects of global assimilated carbon redistribution. 


\section{Conclusions}

Methanotrophic bacteria have the potential to play a significant role in the emerging bioeconomy, serving as biocatalysts for the conversion of natural gas- and anaerobic digestion-derived methane to an array of petrochemical replacements. Additionally, methanotrophs hold great promise as tools for mitigation of greenhouse gas emissions, serving as a critical methane sink in Earth's biogeochemical carbon cycle. However, to realize the full potential of these microbial catalysts, significant improvements in methane conversion efficiency are needed to achieve industrially relevant conversion metrics. To this end, we have demonstrated effective metabolic engineering strategies to increase intracellular acetyl-CoA levels and enhance yield from methane to microbial biomass and biomass-derived lipids via ectopic overexpression of PKT. The resultant biocatalyst demonstrated $>2$-fold biomass and lipid yield enhancement, underscoring the potential for targeted strain engineering strategies, and generating the most carbon-efficient methanotrophic biocatalyst reported to date.

In addition to the widespread applied implications of these findings, a number of fundamental findings have arisen from these data. Notably, the PKT pathway is significantly upregulated when $M$. buryatense is grown with $\mathrm{CH}_{4}$ compared with $\mathrm{CH}_{3} \mathrm{OH}$ as the sole carbon source, suggesting that this organism might possess $\mathrm{CH}_{4}-$ specific sensing mechanisms. Increased protein acetylation markers and significant differential metabolite distribution in the strain overexpressing PKT supports a fundamental role for this enzyme in global metabolic regulation. Given that protein acetylation is a post-translational regulator of several metabolic enzymes ${ }^{36,37}$, it's possible that as yet unidentified global regulatory effects could contribute to the increased pPKTB biomass yield. Future analyses will target further elucidation of the interplay between PKT pathway flux and transcriptional and translational regulation; a more complete understanding of PKT-regulated methanotrophic metabolism will identify additional strain-engineering opportunities, targeting maximal carbon efficiency in these promising biocatalysts.

\section{Acknowledgments}

We thank the US Department of Energy Bioenergy Technologies Office (DOE-BETO) for funding this work via WBS \# 2.3.2.102 with the National Renewable Energy Laboratory. We thank Mary Lidstrom of University of Washington for M. buryatense 5GB1. We would also like to thank Dr. Marina G. Kalyuzhnaya of San Diego State University for helpful discussions and Nalina Desai of Metabolon for technical assistance. 


\section{References}

1. Bousquet, P. et al. Contribution of anthropogenic and natural sources to atmospheric methane variability. Nature 443, 439-443 (2006).

2. Miller, S. M. et al. Anthropogenic emissions of methane in the United States. Proceedings of the National Academy of Sciences 110, 20018-20022 (2013).

3. Conrado, R. J. \& Gonzalez, R. Chemistry. Envisioning the bioconversion of methane to liquid fuels. Science 343, 621-623 (2014).

4. Strong, P. J., Xie, S. \& Clarke, W. P. Methane as a resource: can the methanotrophs add value? Environ. Sci. Technol. 49, 4001-4018 (2015).

5. Hanson, R. S. \& Hanson, T. E. Methanotrophic bacteria. Microbiol. Rev. 60, 439-471 (1996).

6. Anthony, C. The biochemistry of methylotrophs. (Academic Pr, 1982).

7. Gilman, A. et al. Bioreactor performance parameters for an industriallypromising methanotroph Methylomicrobium buryatense 5GB1. Microb. Cell Fact. 14, 1-8 (2015).

8. Henard, C. A. et al. Bioconversion of methane to lactate by an obligate methanotrophic bacterium. Sci Rep 6, 1-9 (2016).

9. Kalyuzhnaya, M. G., Puri, A. W. \& Lidstrom, M. E. Metabolic engineering in methanotrophic bacteria. Metabolic Engineering 29, 142-152 (2015).

10. Puri, A. W. et al. Genetic Tools for the Industrially Promising Methanotroph Methylomicrobium buryatense. Appl. Environ. Microbiol. 81, 1775-1781 (2015).

11. Yan, X., Chu, F., Puri, A. W., Fu, Y. \& Lidstrom, M. E. Electroporation-Based Genetic Manipulation in Type I Methanotrophs. Appl. Environ. Microbiol. 82, 2062-2069 (2016).

12. Fei, Q. et al. Bioconversion of natural gas to liquid fuel: opportunities and challenges. Biotechnol. Adv. 32, 596-614 (2014).

13. Henard, C. A., Freed, E. F. \& Guarnieri, M. T. Phosphoketolase pathway engineering for carbon-efficient biocatalysis. Current Opinion in Biotechnology 36, 183-188 (2015).

14. Rohr, L. M., Teuber, M. \& Meile, L. Phosphoketolase, a neglected enzyme of microbial carbohydrate metabolism. Chimia 56, 270-273 (2002).

15. Stincone, A. et al. The return of metabolism: biochemistry and physiology of the pentose phosphate pathway. Biol Rev Camb Philos Soc (2014). doi:10.1111/brv.12140

16. Rozova, O. N., Khmelenina, V. N., Gavletdinova, J. Z., Mustakhimov, I. I. \& Trotsenko, Y. A. Acetate kinase-an enzyme of the postulated phosphoketolase pathway in Methylomicrobium alcaliphilum 20Z. Antonie Van Leeuwenhoek 108, 965-974 (2015).

17. Xiong, W. et al. Phosphoketolase pathway contributes to carbon metabolism in cyanobacteria. Nat Plants 2, 15187 (2015).

18. Chinen, A., Kozlov, Y. I., Hara, Y., Izui, H. \& Yasueda, H. Innovative metabolic pathway design for efficient l-glutamate production by suppressing $\mathrm{CO} 2$ emission. J Biosci Bioeng 103, 262-269 (2007).

19. Bergman, A., Siewers, V., Nielsen, J. \& Chen, Y. Functional expression and 
evaluation of heterologous phosphoketolases in Saccharomyces cerevisiae. AMB Express 6, 115 (2016).

20. Kocharin, K., Siewers, V. \& Nielsen, J. Improved polyhydroxybutyrate production by Saccharomyces cerevisiae through the use of the phosphoketolase pathway. Biotechnol. Bioeng. 110, 2216-2224 (2013).

21. de Jong, B. W., Shi, S., Siewers, V. \& Nielsen, J. Improved production of fatty acid ethyl esters in Saccharomyces cerevisiae through up-regulation of the ethanol degradation pathway and expression of the heterologous phosphoketolase pathway. Microb. Cell Fact. 13, 39 (2014).

22. Sonderegger, M., Schumperli, M. \& Sauer, U. Metabolic Engineering of a Phosphoketolase Pathway for Pentose Catabolism in Saccharomyces cerevisiae. Appl. Environ. Microbiol. 70, 2892-2897 (2004).

23. Meadows, A. L. et al. Rewriting yeast central carbon metabolism for industrial isoprenoid production. Nature 537, 694-697 (2016).

24. Panagiotou, G. et al. Studies of the production of fungal polyketides in Aspergillus nidulans by using systems biology tools. Appl. Environ. Microbiol. 75, 2212-2220 (2009).

25. Bogorad, I. W., Lin, T.-S. \& Liao, J. C. Synthetic non-oxidative glycolysis enables complete carbon conservation. Nature 502, 693-697 (2013).

26. Glenn, K. \& Smith, K. S. Allosteric regulation of Lactobacillus plantarum xylulose 5-phosphate/fructose 6-phosphate phosphoketolase (Xfp). J. Bacteriol. 197, 1157-1163 (2015).

27. Servinsky, M. D. et al. Arabinose is metabolized via a phosphoketolase pathway in Clostridium acetobutylicum ATCC 824. J Ind Microbiol Biotechnol 39, 1859-1867 (2012).

28. Fu, Z. et al. Exometabolome analysis reveals hypoxia at the up-scaling of a Saccharomyces cerevisiae high-cell density fed-batch biopharmaceutical process. Microb. Cell Fact. 13, 32 (2014).

29. Bogorad, I. W. et al. Building carbon-carbon bonds using a biocatalytic methanol condensation cycle. Proceedings of the National Academy of Sciences 111, 15928-15933 (2014).

30. Kalyuzhnaya, M. G. et al. Highly efficient methane biocatalysis revealed in a methanotrophic bacterium. Nat Commun 4, - (2013).

31. Bothe, H. et al. Heterotrophic bacteria growing in association with Methylococcus capsulatus (Bath) in a single cell protein production process. Appl. Microbiol. Biotechnol. 59, 33-39 (2002).

32. Chistoserdova, L. \& Lidstrom, M. E. in 267-285 (Springer Berlin Heidelberg, 2013). doi:10.1007/978-3-642-30141-4_68

33. la Torre, de, A. et al. Genome-scale metabolic reconstructions and theoretical investigation of methane conversion in Methylomicrobium buryatense strain 5G(B1). Microb. Cell Fact. 14, 188 (2015).

34. Vadali, R. Cofactor engineering of intracellular CoA/acetyl-CoA and its effect on metabolic flux redistribution in Escherichia coli. Metabolic Engineering 6, 133-139 (2004).

35. Malashenko, Y. R., Pirog, T. P., Romanovskaya, V. A., Sokolov, I. G. \& Grinberg, T. A. Search for Methanotrophic Producers of Exopolysaccharides. Applied 
Biochemistry and Microbiology 37, 599-602 (2001).

36. Pisithkul, T., Patel, N. M. \& Amador-Noguez, D. Post-translational

modifications as key regulators of bacterial metabolic fluxes. Curr. Opin. Microbiol. 24, 29-37 (2015).

37. Hu, L. I., Lima, B. P. \& Wolfe, A. J. Bacterial protein acetylation: the dawning of a new age. Mol. Microbiol. 77, 15-21 (2010). 